The static spacetime relative acceleration for general free fall and its possible experimental test

This article has been downloaded from IOPscience. Please scroll down to see the full text article.

1998 Class. Quantum Grav. 151557

(http://iopscience.iop.org/0264-9381/15/6/012)

View the table of contents for this issue, or go to the journal homepage for more

Download details:

IP Address: 200.130.19.138

The article was downloaded on 22/08/2013 at 19:41

Please note that terms and conditions apply. 


\title{
The static spacetime relative acceleration for general free fall and its possible experimental test
}

\author{
F Bunchaft and S Carneiro \\ Instituto de Física, Universidade Federal da Bahia 40210-340, Salvador, BA, Brazil \\ Received 15 July 1997, in final form 27 January 1998
}

\begin{abstract}
Mishra has recently established, using a generic static metric, the relative local proper time 3-acceleration of a test particle in one-dimensional free fall relative to a static reference frame in any static spacetime. In this paper, on the grounds of gravitoelectromagnetism we establish, in a covariant spacetime form, the relative 4-acceleration for general free fall, indicating its canonical representation with its 3-space kinematic content. Then we obtain the relation between this representation and the very well known expression for relative free-fall acceleration in Fermi coordinates. Taking this into account, it is shown that an experiment with relativistic beams in a circular accelerator, modelled by Fermi coordinates, recently proposed by Moliner $e t a l$, can test the covariant result established here, and, therefore, can also verify Mishra's formula. This possibility of experimental verification, besides its intrinsic importance, can answer a recent question by Vigier, related to his recent proposal of derivation of inertial forces.
\end{abstract}

PACS numbers: 0420C, 0480C

\section{Introduction}

Mishra [1] has recently presented some relations (the 'transformation law' and the 'addition law' for accelerations) between kinematic observations on particles in one-dimensional motion in a general relativistic static spacetime, as performed by accelerated observers in this spacetime. From his general relativistic addition law Mishra obtains, in particular, the formula $\vec{a}=\gamma^{-2} \vec{g}$ (see equation (11) in [1]) for the local proper time relative 3-acceleration $\vec{a}$ (Mishra's 'physical acceleration') of a relativistic particle in one-dimensional free fall in any static gravitational field, relative to a preferred nonlinear static reference frame $(\vec{g}$ stands for the acceleration when the 'physical' relative velocity $\vec{v}$ is zero; $\gamma^{-2}=\left(1-v^{2}\right)$ in units in which $c=1$ ).

More recently Vigier has pointed out the convenience of performing a laboratory test to verify this formula. His suggestion comes up in the course of an extensive article [2] dedicated to exposing a possible, non-Machian, solution to the age-old 'unsolved mystery in modern physics' of the origin and nature of inertia. In his proposal, inertial forces arise from the local interaction of a physical vacuum or ether (in Dirac's covariant model) with accelerated particle-like solitons piloted by surrounding wavepackets. For simplicity Vigier considers only the one-dimensional accelerated motion of the solitons in the ether and puts to work some inertial and accelerated observers/frames. Then the previous Mishra's formula, says Vigier, ... 'plays a crucial role in our derivation/interpretation of inertia. It evidently proves that the acceleration due to a pseudo-force (inertial force) and that due to the force of gravity are both decreased by a factor $\left(1-v^{2} / c^{2}\right) \ldots$ ' 
In this paper, we put the problem in a broader context, following the gravitoelectromagnetism (GEM) formalism as outlined by Bini et al [3]. These authors have, in particular, re-obtained, in a coordinate-free form, all the results of Mishra.

Our first step is to extend, in a covariant spacetime form, Mishra's formula to the case of static general free fall. Then we represent the obtained 4-covariant formula in a canonical form in the observer's computational 3-space and proper time. Then, introducing observeradapted Fermi coordinates (FC) we obtain the relations between the canonical representation and the well known general free-fall relative Fermi coordinates acceleration $\vec{a}_{F C}$.

As we will see, $\vec{a}_{F C}$ does not coincide with the relative acceleration in the canonical representation and does not contain Mishra's one-dimensional expression. In contrast, the measurable physics which stems from $\vec{a}_{F C}$ for the one-dimensional free fall is 'strange' and radically different from that which comes from Mishra's law.

Indeed, for $\hat{v}_{F C}=\hat{g}$ it will be $\vec{a}_{F C}=\mathrm{d} \vec{v}_{F C} / \mathrm{d} x_{F C}^{0}=k \vec{g}$, with $k=\left(1-2 v_{F C}^{2}\right)$. Thus, only for $v_{F C}<2^{-1 / 2}$ it will be $k>0$. For $v_{F C}=2^{-1 / 2}$, the particle will follow a FCuniform movement and, when $v_{F C}>2^{-1 / 2}$, it will be $k<0$. Clearly, these kinds of 'anomalies' are absent from Mishra's expression $\dagger$.

Obtaining a connection between the canonical relative 3-acceleration representation of the GEM spacetime formula and Fermi's relative 3-acceleration allows us to reconsider an experiment proposed recently by Moliner et al [5] with almost horizontal relativistic particles in a circular accelerator in order to test $\vec{a}_{F C}$, with Fermi coordinates modelling the experiment. In view of the previously obtained relations between the GEM formulae and $\vec{a}_{F C}$, we show that the realm of the experiment can be considerably enlarged to also test the GEM expressions and their theoretical implications, in particular, Mishra's formula. This, besides being a possible test of general relativity in a terrestrial experiment involving relativistic massive particles, would also be significant as an estimate of Vigier's derivation of inertial forces.

\section{The GEM formula for general free fall}

\subsection{Bini et al's exposition}

Let us start by briefly recalling Bini et al's exposition. Spatial gravitational forces modelled after the electromagnetic 4-force, that is, 'gravitoelectromagnetic forces', rely on the splitting of spacetime by means of a congruence of test observers $(u)$. The decomposition of each tangent space into a local direction along the 4-velocity vector field $u$ of $(u)$ and its orthogonal complement, the local instantaneous $u$-rest space $L R S_{u}$, induces a corresponding coordinate-free decomposition of all spacetime tensors and tensor equations, leading to spatial spacetime tensor fields (any contraction with $u$ gives zero) and spatial equations which represent them, i.e. which 'measure' them. This decomposition is accomplished by $T(u)$ and $P(u)$, the operators of temporal projection and of spatial projection into $L R S_{u}$, respectively, being $P(u) X=X+u[u \cdot X]$. These operators may be identified with suitable mixed second-rank tensors acting by contraction.

Through $P(u)$ there are also introduced spatially projected differential operators: so, from the spacetime covariant derivation operator ${ }^{4} \nabla$, arises the spatial covariant derivative $\nabla(u)=P(u)^{4} \nabla$, the spatial Fermi-Walker derivative $\nabla_{f w}(u)=P(u)^{4} \nabla_{u}$ (which for spatial tensor fields coincides with the spacetime Fermi-Walker derivative along $u$ ), etc.

$\dagger$ Jaffe and Shapiro [4] have already obtained the same 'anomalies' for the spherically symmetric gravitational field in Schwarzschild coordinates. 
Now considering a test particle $(U)$ with 4-velocity $U$, the orthogonal decomposition of $U$ with respect to $u$ defines the relative velocity $v(U, u)$ of $(U)$ with respect to $(u)$ and the associated gamma factor, i.e.

$$
U=\gamma(U, u)[u+v(U, u)]
$$

where

$$
\gamma(U, u)=[1-v(U, u) \cdot v(U, u)]^{-1 / 2}
$$

(a dot represents the inner product of spacetime vectors). $v(U, u)$ is $u$-spatial, since it is the rescaled $u$-spatial projection of $U$, i.e.

$$
v(U, u)=\gamma(U, u)^{-1} P(u) U .
$$

Now let $a(U)=\nabla_{U} U$ be the 4-acceleration of $(U)$ and let $a(U)=\tilde{f}(U)$ be the equation of motion for $U$, where $\tilde{f}(U)$ is the 4-force per unit mass on the test particle $U$. Then the orthogonal decomposition and the spatial projection of the spacetime tensors and of the equation of motion lead to

$$
A(U, u)=\tilde{F}(U, u)
$$

where

$$
A(U, u)=\gamma(U, u)^{-1} P(u) a(U)
$$

(and equivalently for $\tilde{F}(U, u)$ and $\tilde{f}(U))$.

Expressing $A(U, u)$ in terms of the relative momentum per unit mass, $\tilde{p}(U, u)=$ $\gamma(U, u) v(U, u)$, introducing the composite projection map defined by $P(u, U, u)=$ $P(u) P(U) P(u)$, which is an automorphism of $L R S_{u}$, and defining

$$
a_{f w}(U, u)=\gamma(U, u)^{-1} P(u) \nabla_{U} v(U, u)
$$

as the Fermi-Walker relative acceleration of $U$ with respect to $u$, Bini et al have derived the expression

$A(U, u)=-\gamma(U, u)\left[g(u)+H_{f w}(u) v(U, u)\right]+\gamma(U, u) P(u, U, u) a_{f w}(U, u)$.

Here

$$
\begin{aligned}
& g(u)=-a(u) \\
& H_{f w}(u)=-\nabla(u) u=\omega(u)-\theta(u)
\end{aligned}
$$

where $\omega(u)$ and $\theta(u)$ are, respectively, the vorticity (rotation) and expansion tensors of the observers family $(u)$, and a contraction being implied between $H_{f w}(u)$ and $v(U, u)$.

Now, $a_{f w}(U, u)$ can be rewritten as

$$
a_{f w}(U, u)=\frac{\mathrm{D}_{f w}(U, u)}{\mathrm{d} \tau_{U, u}} v(U, u)
$$

where $\mathrm{D}_{f w}(U, u) / \mathrm{d} \tau_{U, u}$ is the Fermi-Walker total spatial covariant derivative along the worldline $U$ expressed in terms of a parametrization corresponding to the sequence of differential proper times of the observers $(u)$ along the worldline $U$, so that

$$
\frac{\mathrm{d} \tau_{U, u}}{\mathrm{~d} \tau_{U}}=\gamma(U, u)
$$

where $\mathrm{d} \tau_{U}$ corresponds to the $U$ proper time parametrization.

Besides, we can also obtain

$$
\frac{\mathrm{D}_{f w}(U, u)}{\mathrm{d} \tau_{U, u}} \tilde{p}(U, u)=\tilde{F}_{f w}^{G}(U, u)+\tilde{F}(U, u)
$$


where

$$
\tilde{F}_{f w}^{G}(U, u)=\gamma(U, u)\left[g(u)+H_{f w}(u) v(U, u)\right] .
$$

This allows us to interpret $\tilde{F}_{f w}^{G}(U, u)$ as the relative spatial gravitational force on $U$ with respect to $u$, with $g(u)$ the gravitoelectric vector-force field and $H_{f w}(U, u)$ the gravitomagnetic one. (For a Minkowski spacetime in which $(u)$ is an inertial observer, $\tilde{F}_{f w}^{G}(U, u)$ is zero.)

\subsection{Covariant extension of Mishra's result}

We will now extend Mishra's result, obtaining the expression for the relative 4-acceleration in the case of general free fall.

Let us begin by observing that, in our case, the test particle $U$ is free, so the 4acceleration $a(U)$ is null. Thus we have $A(U, u)=0$.

Also, the spacetime is static and $(u)$ is the preferred local reference frame, that is, $u$ is the direction of a timelike Killing vector field, which implies that $\theta(u)=\omega(u)=0$. So, $H_{f w}(u)=0$ and (7) reduces to

$$
P(u) P(U) P(u) a_{f w}(U, u)=g(u) .
$$

Now taking into account that, by definition, $a_{f w}(U, u)$ and $v(U, u)$ are $u$-spatial and using the orthogonal decomposition (1) of $U$, the successive projections (14) lead to

$$
a_{f w}(U, u)+\gamma^{2}\left[a_{f w}(U, u) \cdot v(U, u)\right] v(U, u)=g(u) .
$$

Now, multiplying this equation by $v(U, u)$ and considering the definition (2) of $\gamma(U, u)$, we obtain

$$
\gamma^{2}\left[a_{f w}(U, u) \cdot v(U, u)\right] v(U, u)=g(u) \cdot v(U, u)
$$

or, finally,

$$
a_{f w}(U, u)=g(u)-[g(u) \cdot v(U, u)] v(U, u)
$$

which is the 4-covariant GEM expression for general free fall in a static spacetime†.

We can immediately verify that, for $\hat{v}(U, u)=\hat{g}(u)$, it results that

$$
a_{f w}(U, u)=\gamma^{-2} g(u),
$$

which is the spacetime covariant expression of Mishra's result for the one-dimensional free fall.

\section{The canonical representation}

Equation (17) as well as (18) previously derived are general covariant equations for the free-fall 4-acceleration relative to a preferred static reference frame where the observers constitute a timelike Killing vector field, always present in some open submanifold of any stationary spacetime. Since our generic spacetime metric is not only stationary but, more than that, static, the observers local rest space constitutes a spatial-like slicing orthogonal to the observers spacetime threading.

Thus we can complete the spacetime threading to an observer-adapted frame, that is, any frame $\left\{e_{\alpha}\right\}, \alpha=0,1,2,3$, such that $e_{0}$ is along the observer 4-velocity $u$ and the spatial

$\dagger$ This result can also be obtained directly by using the expression of $[P(u) P(U) P(u)]$ as given in line 1 of table 1 of [3], or, alternatively, by solving (14) for $a_{f w}(U, u)$, using the expression of $[P(u) P(U) P(u)]^{-1}$ as given in line 2 of the table, as suggested by an anonymous referee. 
frame $\left\{e_{i}\right\}, i=1,2,3$, spans the local rest space at each point along $u$. Besides, the spatial frame is Fermi-Walker transported along $u$, which assures that it remains orthogonal (and even orthonormal if we choose so). Finally, we take for our present purposes the frame basis to be a coordinate one, $\left\{x^{\alpha}\right\}$ being the local coordinates adapted to the frame.

Under these conditions, the general theory shows that the algebra of stationary spatial tensors is isomorphic to the tensor algebra of the computational 3-space equipped with the time-independent projected spatial metric $\gamma_{i j}$ expressible in the local adapted coordinates $[3,6]$.

Furthermore, since the $g_{\alpha \beta}$ metric is static, any spatial-like orthogonal slice can be taken as the computational space and $\gamma_{i j}=g_{i j}$. Besides, the spatial operators of static spatial fields reduce to the correspondent operators defined with respect to $\gamma_{i j}$. Hereafter a nonlinear static reference frame will always be considered equipped with the above-defined structures.

Now, returning to the 4-covariant GEM equation referred to such a static reference frame, $g(u)=-\nabla_{u} u$ and, since $u$ is a unit vector, $g(u)$ is spatial and, by definition, $a_{f w}(U, u)$ and $v(U, u)$ also are. Then the projected equation can be noted in the 3-space vector notation

$$
\overrightarrow{\tilde{a}}=\vec{g}-\vec{g} \cdot \overrightarrow{\tilde{v}} \overrightarrow{\tilde{v}}
$$

where $a_{f w}(U, u)=(0, \overrightarrow{\tilde{a}}), g(u)=(0, \vec{g}), v(U, u)=(0, \overrightarrow{\tilde{v}})$ and the spatial inner product can be considered as arising from

$$
X \cdot{ }_{u} Y=P(u)_{\alpha \beta} X^{\alpha} Y^{\beta}=g_{i j} X^{i} Y^{j}=\gamma_{i j} x^{i} y^{j}
$$

for any pair of spatial vector fields $X=(0, \vec{x})$ and $Y=(0, \vec{y})$. The projected equation (19), for its naturalness, will be said to be the canonical representation of the covariant GEM expression (17).

The generic relation between the projected variables $\overrightarrow{\tilde{v}}$ and $\overrightarrow{\tilde{a}}$ in the canonical representation and the relative 3-geometric kinematic variables is also given by the general theory $[3,6]$, but it will be worthwhile for our immediate purposes to unfold it here directly.

Then let $x_{U}^{\alpha}$ be the coordinates of some particle worldline with 4-velocity $U$. So $\mathcal{U}^{\alpha}=\mathrm{d} x_{U}^{\alpha} / \mathrm{d} x^{0}=\dot{x}_{U}^{\alpha}$ will denote the coordinate components of the coordinate velocity of the $U$ worldline, whose components are

$$
U^{\alpha}=\frac{\mathrm{d} x_{U}^{\alpha}}{\mathrm{d} \tau_{U}}=\dot{x}_{U}^{\alpha}\left(\frac{\mathrm{d} x_{U}^{0}}{\mathrm{~d} \tau_{U}}\right)=\Gamma_{x^{\alpha}}(U, u) \dot{x}_{U}^{\alpha}
$$

where

$$
\frac{\mathrm{d} x_{U}^{0}}{\mathrm{~d} \tau_{U}}=\left(g_{\alpha \beta} \dot{x}_{U}^{\alpha} \dot{x}_{U}^{\beta}\right)^{-1 / 2}=\left(\mathcal{U}_{\alpha} \mathcal{U}^{\alpha}\right)^{-1 / 2} \equiv \Gamma_{x^{\alpha}}(U, u)
$$

is the $x^{\alpha}$-coordinate Lorentz factor.

Let us now denote by $v^{i}$ the coordinates of a 3 -vector $\vec{v}$ defined by

$$
v^{i}=\frac{\mathrm{d} x_{U}^{i}}{\mathrm{~d} \tau_{U, u}}=\dot{x}_{U}^{i} g_{00}^{-1 / 2}
$$

since $\mathrm{d} x_{U}^{0} / \mathrm{d} \tau_{U, u}=g_{00}^{-1 / 2}$ in the static metric expressed in $x^{\alpha}$-coordinates.

Note that this definition implies that

$$
v^{2}=\left(\frac{\mathrm{d} l}{\mathrm{~d} \tau_{U, u}}\right)^{2}
$$


where $\mathrm{d} l^{2}=g_{i j} \mathrm{~d} x_{U}^{i} \mathrm{~d} x_{U}^{j}$. So $\vec{v}$ is the 3-velocity of the $U$-particle measured by the $u$ observer at rest in the same place as the $U$-particle, in the proper time of the observer, to be called hereafter the local proper velocity of the particle $U$.

Then, denoting $\gamma^{*}(U, u)=\left[1-v^{2}\right]^{-1 / 2}$, we have

$$
\Gamma_{x^{\alpha}}(U, u)=g_{00}^{-1 / 2}\left[1-g_{i j} v^{i} v^{j}\right]^{-1 / 2}=g_{00}^{-1 / 2} \gamma^{*}(U, u) .
$$

Thus $\gamma^{*}(U, u)=\gamma(U, u)=\mathrm{d} \tau_{U, u} / \mathrm{d} \tau_{U}$, as defined in (11), and

$$
\begin{aligned}
& U^{i}=\gamma(U, u) v^{i} \\
& U^{0}=\gamma(U, u) g_{00}^{-1 / 2} .
\end{aligned}
$$

Therefore,

$$
v^{i}=\gamma^{-1}(U, u) U^{i}=\gamma^{-1}(U, u)[P(u) U]^{i}
$$

which, by definition of $v(U, u)$ (see equation (3)), leads to $v^{i}=v^{i}(U, u)=(0, \overrightarrow{\tilde{v}})^{i}$, thus identifying $\overrightarrow{\tilde{v}}$ with $\vec{v}$, that is, with the local 3-proper-velocity of the particle $U$ relative to the stationary observer $u$.

Let us consider now the kinematic meaning of the spatial projection $\overrightarrow{\tilde{a}}$ of $a_{f w}(U, u)$ in the spatial geometry associated to the static reference frame. From definition (10) comes

$a_{f w}(U, u)=P(u) \frac{\mathrm{D}}{\mathrm{d} \tau_{U, u}}\left[\gamma^{-1}(U, u) P(u) U\right]=P(u) \frac{\mathrm{D}}{\mathrm{d} \tau_{U, u}}(0, \overrightarrow{\tilde{v}})=(0, \overrightarrow{\tilde{a}})$

so that we can identify the projected acceleration $\overrightarrow{\tilde{a}}$ with

$$
(\vec{a})^{i}=\left(\frac{{ }^{3} \mathrm{D} \vec{v}}{\mathrm{~d} \tau_{U, u}}\right)^{i}=\left(\frac{\mathrm{d} \vec{v}}{\mathrm{~d} \tau_{U, u}}\right)^{i}+\Gamma_{j k}^{i} v^{j} v^{k}
$$

where $\Gamma_{j k}^{i}$ are the components of the Riemannian connection associated to the 3-metric $g_{j k}$. That is, we can identify $\overrightarrow{\tilde{a}}$ with $\vec{a}$, the local 3-proper-acceleration of the particle $U$ relative to the stationary observer $u$.

So, we have shown that the canonical representation of the covariant GEM formula can be identified with the expression

$$
\vec{a}=\vec{g}-\vec{g} \cdot \vec{v} \vec{v}
$$

where $\vec{a}$ and $\vec{v}$ have now a precise 3 -geometric kinematic content.

Let us observe that an expression like (31) has been presented recently by Mould [7] for uniformly accelerated frames in the flat spacetime, starting from the specific metric to obtain the coordinate acceleration and changing then to the suitable local proper observers (see equation (8.45) in [7]). So, the present GEM derivation can be said to extend Mould's expression to any static spacetime and Mishra's result to general free fall.

\section{The GEM formulae and the kinematic variables in Fermi coordinates}

Let us consider Fermi coordinates adapted to a stationary observer modelled by a worldline $u$ with covariant 4-acceleration $a(u)=-g(u)$. As these coordinates are suitable to model terrestrial experiments like the one considered in the next section, let us now try to connect the GEM formulae, through the canonical representation derived previously, with the kinematic variables in these particular coordinates.

For this purpose we need to establish suitable relations between the local kinematic variables, in the canonical representation, and the adapted Fermi coordinates kinematic variables. (Clearly, in this context, a preliminary assumption must be the Fermi-Walker 
transport of the Fermi spatial frame, in order to assure orthonormality and 'non-rotation' of the frame.)

Locally, in Fermi coordinates, the spacetime metric will be, as is well known (see equation (13.71) in [8])

$\mathrm{d} s^{2}=\left(1-2 g_{i} x_{F C}^{i}\right)\left(\mathrm{d} x_{F C}^{0}\right)^{2}-\delta_{i j} \mathrm{~d} x_{F C}^{i} \mathrm{~d} x_{F C}^{j}+\mathrm{O}\left(\left|x_{F C}^{i}\right|^{2}\right) \mathrm{d} x_{F C}^{\alpha} \mathrm{d} x_{F C}^{\beta}$

where $\alpha, \beta=0,1,2,3 ; i, j=1,2,3 ; g(u)=(0, \vec{g}) ; g_{i}=(\vec{g})_{i}$. Stationarity implies that $\vec{g}$ does not depend on $x_{F C}^{0}$.

This means that our observer has been immersed in a family of local stationary observers $(u)$, whose local rest spaces integrate to a spacelike hypersurface which is locally flat at this order of approximation, but whose coordinate clocks are all paced by our observer at the origin. Let us recall that, in the previous GEM equations, all the local observers at rest in the frame reparametrize any geodesic worldline $U$ of a particle in free fall by their own local proper time $\tau_{U, u}$ (not by the coordinate time $x^{0}$ or by the proper time $\tau_{U}$ of $U$ ).

Now, let us apply (23) and (30) to the adapted Fermi coordinates. With the notation $v_{F C}^{i}=\dot{x}^{i}, a_{F C}^{i}=\ddot{x}^{i}$, one has

$$
\begin{aligned}
& \vec{v}=g_{00}^{-1 / 2 \dot{\vec{x}}}=\left(1-2 g_{i} x_{F C}^{i}\right)^{-1 / 2} \vec{v}_{F C} \\
& \vec{a}=\frac{{ }^{3} \mathrm{D} \vec{v}}{\mathrm{~d} \tau_{U, u}}=\frac{\mathrm{d} \vec{v}}{\mathrm{~d} \tau_{U, u}}
\end{aligned}
$$

since the spatial 3-metric $g_{i j}=\delta_{i j}$ is flat. Then, from $\mathrm{d} \tau_{U, u}=g_{00}^{1 / 2} \mathrm{~d} x_{F C}^{0}$ it will be

$\vec{a}=g_{00}^{-1 / 2} \frac{\mathrm{d}}{\mathrm{d} x_{F C}^{0}}\left(g_{00}^{-1 / 2} \vec{v}_{F C}\right)=\left(1-2 g_{i} x_{F C}^{i}\right)^{-1} \vec{a}_{F C}+\left(1-2 g_{i} x_{F C}^{i}\right)^{-2} g_{j} v_{F C}^{j} \vec{v}_{F C}$.

For the observer at the origin we have

$$
\begin{aligned}
& \vec{v}=\vec{v}_{F C} \\
& \vec{a}=\vec{a}_{F C}+\vec{g} \cdot \vec{v}_{F C} \vec{v}_{F C}
\end{aligned}
$$

which establishes the relations between the canonical kinematic variables and the Fermi coordinate ones.

On the other hand, $\vec{a}$ is expressed by (31), so that, in view of (36),

$$
\vec{a}=\vec{g}-\vec{g} \cdot \vec{v}_{F C} \vec{v}_{F C} .
$$

The consistency of the pair of equations (37) and (38) for $\vec{a}$ can be verified by obtaining from them the very well known expression

$$
\vec{a}_{F C}=\vec{g}-2 \vec{g} \cdot \vec{v}_{F C} \vec{v}_{F C}
$$

for the Fermi coordinate acceleration, which is usually obtained directly by a very distinct derivation (see equation (13.75) of [8]).

Note the essential theoretical difference between $\vec{a}$ and $\vec{a}_{F C}$ : $\vec{a}$ is constructed in a coordinate-free manner and so is invariant; the change of the temporal parametrization (from the observer's proper time to the Fermi coordinate time) of the test particle worldline when we go from the canonical representation to the Fermi coordinates produces the variation $\vec{a}-\vec{a}_{F C}=\vec{g} \cdot \vec{v}_{F C} \vec{v}_{F C} \neq 0$, when $\vec{g} \cdot \vec{v}_{F C} \neq 0$ (or $g_{i} x_{F C}^{i} \neq 0$ ), since clocks at different heights beat at different rates (otherwise, $\vec{a}=\vec{a}_{F C}=\vec{g}$ ). (Concerning spatial coordinates, let us recall that the Fermi ones measure proper distances, in this order of approximation.)

Clearly, from the expression for $\vec{a}_{F C}$, changing from coordinate time to local proper time, it would be possible to derive $\vec{a}$, but this would hide the canonical content of $\vec{a}$ and of equation (31) as the canonical 3-space representations of the spacetime-covariant FermiWalker relative acceleration $a_{f w}(U, u)$ and of the covariant equation (17), respectively, that is, the covariant content of the problem. 


\section{A possible test for the GEM expressions}

Moliner et al [5] have recently suggested a possible way to test the Fermi coordinate acceleration expression. In their suggestion, such a test is to be performed in a circular accelerator where a charged particle moves under the influence of suitable electric and magnetic fields, besides, of course, the Earth's gravitational one.

Then, equation (39) is extended to give

$$
\vec{a}_{F C}=\vec{g}-2 \vec{g} \cdot \vec{v}_{F C} \vec{v}_{F C}+\frac{e}{m \Gamma_{F C}(U, u)}\left(\vec{E}+\vec{v}_{F C} \times \vec{H}-\vec{E} \cdot \vec{v}_{F C} \vec{v}_{F C}\right)
$$

where $\vec{E}$ and $\vec{H}$ are the electric and magnetic fields, $e$ is the charge, $m$ is the mass and $\Gamma_{F C}(U, u)=\left(1-v_{F C}^{2}\right)^{-1 / 2}$ is the Fermi coordinate Lorentz factor.

Defining

$$
\vec{E}_{p}=\vec{E}+\frac{m \Gamma_{F C}(U, u)}{e} \vec{g}
$$

equation (40) can be rewritten in the form

$$
\vec{a}_{F C}=\frac{e}{m \Gamma_{F C}(U, u)}\left(\vec{E}_{p}+\vec{v}_{F C} \times \vec{H}-\vec{E}_{p} \cdot \vec{v}_{F C} \vec{v}_{F C}\right)-\vec{g} \cdot \vec{v}_{F C} \vec{v}_{F C}
$$

where $-\left(m \Gamma_{F C}(U, u) / e\right) \vec{g}$ is the electric field necessary to prevent the particle falling down.

Taking for $\vec{H}$ a uniform magnetic field and for $\vec{E}_{p}$ a periodic electric field, both in the $\hat{g}$-direction, the term $-\vec{g} \cdot \vec{v}_{F C} \vec{v}_{F C}$ leads to a measurable horizontal drift of the trajectory, due to a resonance effect arising from making the frequency of $\vec{E}_{p}$ equal to the Larmor frequency of the particle in the magnetic field.

If $\vec{E}_{p}=0$, the particle movement will be strictly horizontal and circular, since then $\vec{E}$ would only prevent it following down. Switching the additional electric field $\vec{E}_{p}$, a vertical periodic component is summed up to the movement and a resonance effect can arise. The final result is that the trajectory of the particle projected in the horizontal plane is now a drifting circle. The final vertical velocity comes from $\vec{E}_{p}$ and from the gravitational acceleration arising from the term $\vec{g} \cdot \vec{v}_{F C} \vec{v}_{F C}$. This velocity also contributes to the horizontal component of the particle acceleration through the horizontal part of this same term.

In this way it seems possible to verify experimentally the Fermi coordinate acceleration. Remembering that $\vec{a}_{F C}$ can be correlated, through the canonical representation, to the GEM formula for general free fall and that the latter contains Mishra's result, we are led to reconsider Moliner et al's experiment as a possible way to test both these expressions.

Surely, the following question can be posed: using a circular accelerator in Moliner et al's experiment, why is it that we are obliged to work (and measure) $\vec{a}_{F C}$ instead of working with and measuring $\vec{a}$ directly? The situation is as follows.

(i) In the canonical representation, the trajectory of the particle is time-parametrized by the proper time clock of the observer at rest at the spatial position of the particle. At each position, $\vec{a}$ is local (locally measured), since it refers to an arbitrary small spatial neighbourhood during an arbitrary small interval of time. From the local point of view, all the observers are mutually independent, none is preferred and each one measures $\vec{a}$ at its position (in an arbitrary small neighbourhood). But one cannot characterize a circular trajectory in such a neighbourhood.

(ii) To characterize (to measure) a circular trajectory in the accelerator one must refer to fixed, finite, spatial parameters (e.g. the radius, Cartesian coordinates, etc), which cannot be captured (measured) in such arbitrary small neighbourhoods, so one will necessarily be led to define non-local simultaneity (i.e. Einstein's synchronization plus a common rate of the finitely separated clocks); 
(iii) If the accelerator experiment deals only with strictly horizontal circular trajectories, then all the proper time clocks at rest at the same height are equivalent (they beat at the same rate), so the finitely separated clocks are already naturally coordinated and there is no problem: since then $g_{i} x_{F C}^{i}=0$, i.e. $\vec{g} \cdot \vec{v}_{F C}=0$, one will have (measure) strictly $\vec{a}=\vec{a}_{F C}=\vec{g}$ and no drift. But this implies only the first half of Moliner et al's experiment.

(iv) To reach the entire scope of the experiment, the trajectory of the test particle, by necessity, must be strictly non-horizontal: in fact, it is made to oscillate vertically with respect to the horizontal circumference of reference, so one no longer has a natural time coordinization between the clocks at different heights. But, as we have seen in (ii), such a coordinization is unavoidable for a (global) characterization of the trajectory (besides, the drift is also a non-local effect to be measured). So the observers at different heights made a gentleman's agreement to pace their clocks by the clock at the reference level and, with this agreement, what their devise will measure will be $\vec{a}_{F C}$ (which differs from $\vec{a}$ by $\vec{g} \cdot \vec{v}_{F C} \vec{v}_{F C}$ ) and a non-null drift of the circular horizontal projection of the particle trajectory.

Note that if one tries to reconstruct the theoretical scheme of Moliner et al's experiment (that is, equations (40)-(42), etc) by using $\vec{a}$ directly instead of $\vec{a}_{F C}$, then the term $\vec{g} \cdot \vec{v}_{F C} \vec{v}_{F C}$ automatically disappears from (42) and the theoretical scheme becomes vacuous. So the scheme is consistent just with the empirical, observable, content referred above in (i) and (ii).

In the face of this fact that Moliner et al's experiment does not test the GEM expression or Mishra's result directly, we must re-examine to what extent the physical content effectively involved in the test really includes them.

The following reasoning can be done.

(i) In the general GEM formula, and in its canonical representation, $a_{f w}$ (respectively $\vec{a}$ ) is a sum of two parcels, one according to $\hat{g}$ and the other according to $\hat{v}$. Its reduction to Mishra's expression comes for $\hat{v}=\hat{g}$, i.e. is made up of the contributions of both these parcels. So, any experiment able to verify separately each parcel in $a_{f w}$ (respectively $\vec{a})$ confirms the entire general formula and, in particular, Mishra's expression.

(ii) The comparison between $\vec{a}$ and $\vec{a}_{F C}$ (equations (31) and (39)) shows that each parcel of $a_{f w}$ (respectively $\vec{a}$ ) corresponds to the respective parcel of $\vec{a}_{F C}$. So, any experiment able to verify separately both parcels of $\vec{a}_{F C}$ implies the same kind of verification for $a_{f w}$ (respectively $\vec{a}$ ) (and reciprocally) and so confirms the GEM expression and, consequently, Mishra's equation.

(iii) Fortunately, Moliner et al's experiment tests, separately, each parcel of $\vec{a}_{F C}$ : the measurement of the electric field necessary to avoid the charge falling down during the circular movement tests the parcel according to $\hat{g}$; and the measurement of the horizontal drift velocity tests the parcel according to $\hat{v}$.

Thus we can conclude that, if the experiment gives both the predicted electric field and the forseen drift velocity, it will confirm the generic static GEM expressions for general free fall and, in particular, Mishra's one-dimensional result. Besides, it will favour the soundness of Vigier's solution on the nature of inertial forces.

\section{Acknowledgments}

We would like to thank Drs M Portilla and B M Pimentel for interesting discussions. We are also indebted to the anonymous referees for their constructive suggestions. 


\section{References}

[1] Mishra L 1994 Class. Quantum Grav. 11 L97

[2] Vigier J P 1995 Found. Phys. 251461

[3] Bini D, Carini P and Jantsen R T 1995 Class. Quantum Grav. 122549

[4] Jaffe J and Shapiro I I 1972 Phys. Rev. D 6405

[5] Moliner I, Portilla M and Vives O 1995 Phys. Rev. D 521302

[6] Landau L D and Lifshitz E M 1983 The Classical Theory of Fields (Oxford: Pergamon)

[7] Mould R A 1995 Basic Relativity (Berlin: Springer)

[8] Misner C W, Thorne K S and Wheeler J A 1973 Gravitation (San Francisco, CA: Freeman) 